\title{
An ultra-sensitive and wide measuring range pressure sensor with paper-based CNT film/interdigitated structure
}

\author{
Chao Wang, Xiaojuan Hou, Min Cui, Junbin Yu, Xueming Fan, Jichao Qian, Jian He, \\ Wenping Geng, Jiliang $\mathrm{Mu}$ and Xiujian Chou*
}

\begin{abstract}
Flexible pressure sensors have attracted great attention due to their potential in the wearable devices market and in particular in human-machine interactive interfaces. Pressure sensors with high sensitivity, wide measurement range, and low-cost are now highly desired for such practical applications. In the present investigation, an ultrasensitive pressure sensor with wide measurement range has been successfully fabricated. Carbon nanotubes (CNTs) (uniformly sprayed on the surface of paper) comprise the sensitivity material, while lithographed interdigital electrodes comprise the substrate. Due to the synergistic effects of CNT's high specific surface area, paper's porous structure, interdigital electrodes' efficient contact with CNT, our pressure sensor realizes a wide measurement range from 0 to $140 \mathrm{kPa}$ and exhibits excellent stability through 15,000 cycles of testing. For the paper-based CNT film/interdigitated structure (PCI) pressure sensor, the connection area between the sensitive material and interdigital electrodes dominates in the lowpressure region, while internal change within the sensitive materials plays the leading role in the high-pressure region. Additionally, the PCI pressure sensor not only displays a high sensitivity of $2.72 \mathrm{kPa}^{-1}$ (up to $35 \mathrm{kPa}$ ) but also can detect low pressures, such as that exerted by a resting mung bean (about $8 \mathrm{~Pa}$ ). When attached to the surface of a human body, the pressure sensor can monitor physiological signals, such as wrist movement, pulse beats, or movement of throat muscles. Furthermore, the pressure sensor array can identify the spatial pressure distribution, with promising applications in humanmachine interactive interfaces.
\end{abstract}

Keywords: carbon nanotube, interdigital structure, pressure sensors, paper-based carbon nanotube (CNT) film, ultrabroad measuring range

\section{INTRODUCTION}

The rapid development of smart electric devices and wearable devices like flexible self-power supply systems $[1,2]$, flexible sensors [3-5] and flexible displays [6], has generated considerable excitement. In particular, flexible sensors have attracted significant attention due to their promising applications in electronic skin, wearable health monitoring devices, and human body posture detection systems $[7,8]$. On the basis of diverse sensing mechanisms, flexible sensors can be divided into piezoelectric $[9,10]$, capacitive $[11-13]$, triboelectric [14-16], and piezoresistive [17-19] sensors. In particular, piezoresistive sensors, which can transform a variety of pressure signals into a variety of resistance signals, show key advantages, such as high accuracy, simple signal collection and economical manufacturing. In previous work [20-22], many researchers have focused on increasing pressure sensor sensitivity, rather than on increasing the pressure range of an individual sensor. In order to fully realize human body monitoring including pulse detection, voice recognition, wrist movement, and so on, the flexible pressure sensors need to possess not only a high sensitivity but also a wide pressure range [3-5], from a few $\mathrm{Pa}$ to tens of thousands of $\mathrm{Pa}$ [23-25].

Sensitive materials and structures are the two vital factors affecting the performance of pressure sensors. Generally, sensitive materials such as graphene [26-28], carbon nanotubes (CNTs) [20-22], metal nanoparticles $[29,30]$, conductive nanowires [31-33], and organic polymers [34] are used in flexible sensors because they can be easily attached to the surfaces of flexible substrates or mixed into stretchable polymer. Among these materials, CNT has attracted a great deal of interest due to its

Science and Technology on Electronic Test and Measurement Laboratory, School of Instrument and Electronics, North University of China, Taiyuan 030051, China

" Corresponding author (email: chouxiujian@nuc.edu.cn) 
remarkable electronic properties [35,36]. Furthermore, CNT can directly form new conductive paths when subjected to an external force, since its inherently onedimensional (1D) structure endows a high specific surface area. Another essential factor affecting the performance of pressure sensors is the structure, which can be divided into the following three types. The first structure is a mixture of conductive materials and a polymer such as polydimethylsiloxane (PDMS) [37]. The second structure is comprised of conductive materials attached to a rough PDMS surface: micro-pyramid arrays [38], micro-dome arrays [5], silk fabric microstructures [21], or leaf microstructures [39]. The third structure is a combination of sensitive materials with foam, cotton thread, or other fabrics, which can directly form 3D porous, sensitive units. This third structure has achieved key advantages of low cost, simple manufacturing, and environment friendliness [40,41]. Additionally, researchers have proposed and designed numerous methods for achieving high sensitivity $\left(>1 \mathrm{kPa}^{-1}\right)$ and expanding sensor measurement range $(>10 \mathrm{kPa})[42,43]$. For example, Cao et al. [20] reported a high sensitivity $\left(3.26 \mathrm{kPa}^{-1}\right)$ pressure sensor with a measuring range of 0 to about $2.5 \mathrm{kPa}$, produced by spraying single-walled CNTs on a micro-pyramidal PDMS structure. Bae et al. [5] combined graphene and PDMS with a micro-dome array structure to make a remarkably sensitive $\left(14 \mathrm{kPa}^{-1}\right)$ pressure sensor with a wide range from 0 to $12 \mathrm{kPa}$. Shi et al. [4] proposed to use leaf microstructure and graphene to expand the sensor's measurement range up to $25 \mathrm{kPa}$ with a sensitivity of $1.2 \mathrm{kPa}^{-1}$. However, none of these sensors can maintain good sensitivity and broad measurement range at the same time. Hence, narrow measurement range, low sensitivity, and complicated processes currently limit the application of pressure sensors in wearable devices.

In the present work, we successfully prepared a pressure sensor composed of paper-based CNT film and interdigitated structure that simultaneously achieved high sensitivity and wide measurement range. When CNT solution is uniformly sprayed on wrinkled paper, CNT evenly adheres to fibers of the paper. New conductive paths can be formed by three methods, including CNT deformations of high specific surface area, paper fibers contacted with each other, and paper-based CNT films contacted with interdigitated electrodes. When an ultralow pressure acts on the paper-based CNT film/interdigital (PCI) pressure sensor, a subtle deformation emerges in the pressure-sensitive material and increases the connecting area between the pressure sensor material and the metal electrodes. When a high pressure is applied, internal change within the sensitive material plays a leading role in the change of signal. Even after 15,000 testing cycles, the PCI pressure sensor shows good stability and an ultra-wide measurement range from 0 to $140 \mathrm{kPa}$. At the same time, it shows a sensitivity of $2.72 \mathrm{kPa}^{-1}$ for pressures ranging from 0 to about $35 \mathrm{kPa}$, and a sensitivity of $0.21 \mathrm{kPa}^{-1}$ for pressures ranging from 35 to about $140 \mathrm{kPa}$. The sensor can register wrist pulse measurement and voice recognition, sensing and displaying numerous waveform signals. In addition, this pressure sensor array has been applied to mapping spatial dynamic and static pressure distributions.

\section{EXPERIMENTAL SECTION}

\section{Preparation of PDMS film}

The PDMS matrix and the curing agent (Dow Corning Sylgard 184) (mass ratio 10:1) were fully mixed for at least 20 min by mechanical stirring. Next, the mixture was stayed in a vacuum box for 15 min to completely remove bubbles. Subsequently, the PDMS mixture was spincoated onto silicon at $1000 \mathrm{rpm}$ for $10 \mathrm{~s}$ and solidified at $80^{\circ} \mathrm{C}$ for $40 \mathrm{~min}$. Finally, after the PDMS film was carefully peeled off, a suitable size of PDMS film was fabricated by cutting.

\section{Preparation of PI film with electrode structure}

First, the polyimide (PI) film was ultrasonically cleaned with acetone, alcohol and deionized water. The photoresist (Suzhou RDMICRO Materials Tech Co. Ltd., Photoresist 4620) was spin-coated on the surface of PI film at $3000 \mathrm{rpm}$ and solidified at $100^{\circ} \mathrm{C}$ for $1 \mathrm{~min}$. Patterns were then lithographed on the photoresist with a photolithographic machine; expired photoresist was washed using developer solution (Merck Electronic Materials (Suzhou) Co. Ltd., AZ400K Developer). Finally, the copper was deposited on the PI film by magnetron sputtering instrument (DENTON VACUUM Explorer) and then the film was deposited in acetone to remove the copper deposited on the photoresist. The method produced a PI film with electrodes of interdigitated structure.

\section{Preparation of paper-based CNT film}

At a mass ratio 20:1, CNTs (Suzhou TANFENG Materials Tech Co. Ltd., TF-24111) and polyvinyl pyrrolidone (PVP) were dispersed in deionized water by ultrasonic dispersion for $1 \mathrm{~h}$. Then, the well-dispersed CNT solution was uniformly sprayed on paper. Finally, the paper-based CNT film was placed on the drying table (LabTech $\mathrm{EH} 20 \mathrm{~B}$ ) at $70^{\circ} \mathrm{C}$ for $15 \mathrm{~min}$. 


\section{Preparation of the pressure sensor}

The PDMS film was placed on the top of the paper-based CNT film, while the PI film with electrodes of interdigitated structure was placed below. After the two conductive aluminum tapes and the interdigital electrodes were placed together, the sensor was protected on both sides with Kapton tape (3M).

\section{Pressure sensor measurement}

The morphology of the material surface was investigated using a scanning electron microscope (SEM) (ZEISS EVO18). The CNT X-ray diffraction (XRD) pattern was acquired using a PANalytical-empyrean X-ray diffractometer ( $\mathrm{Cu}-\mathrm{K}$ radiation). The CNT Raman spectrum was obtained using a Raman spectrometer (Cobolt 04-01 laser at $514.5 \mathrm{~nm}$ ). Pressure was applied by a linear force device. The current-time $(I-t)$ curves and current-voltage $(I-V)$ curves were measured by the semiconductor parameter analyzer (Keithley 4200-SCS).

\section{Participants}

The volunteer who participated in human testing gave his informed consent.

\section{RESULTS AND DISCUSSION}

The process for manufacturing the pressure sensor is shown in Fig. 1a, including packaging, electrode, and piezoresistive material fabrication. The process for preparing the paper-based CNT (PBC) film as a piezoresistive material is simple and efficient. First, evenly dispersed CNT solution was prepared by the ultrasonic method and dripped onto the crinkled paper at equal intervals and constant speed. Due to the good wettability of paper fibers and their porous structure, the above method results in a uniform PBC film in which CNTs are evenly distributed along the fiber surface. Next, the PBC film was dried by using a heating table and then cut to a suitable size. Fabricating the interdigitated electrodes with an accurate shape and good performance on PI film was mainly achieved through two steps. Firstly, an accurate pattern of interdigitated electrodes was formed on the PI film by lithography technology. Secondly, $\mathrm{Cu}$ electrode was deposited on the processed PI film, and the redundant structure was washed in acetone afterwards. In order to construct the PCI pressure sensor, a PDMS film was used to encapsulate the PBC and PI film together. Fig. $1 \mathrm{~b}$ illustrates the working mechanism of the $\mathrm{PBC}$ pressure sensor, which relies on three mechanisms for altering the resistance. When a force is applied, CNTs in the same fiber touch each other, different conductive fibers filled with CNTs adjoin each other, and the improved contact area between $\mathrm{PBC}$ film and electrode results in the multiplication of conductive paths. Fig. 1c shows that as the external forces are released, the re- a
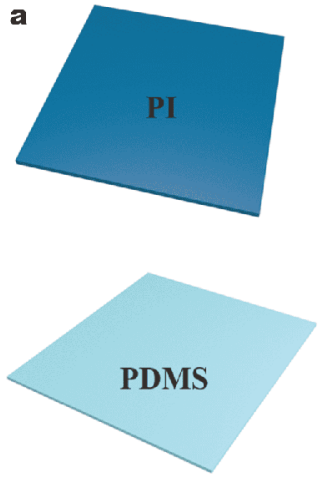

b

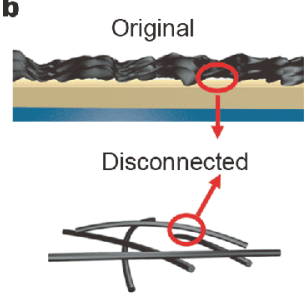

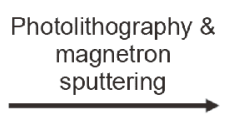
sputtering
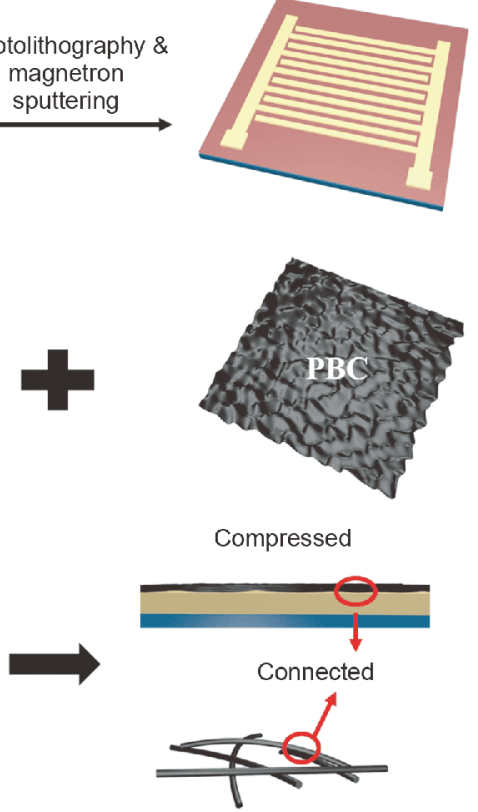

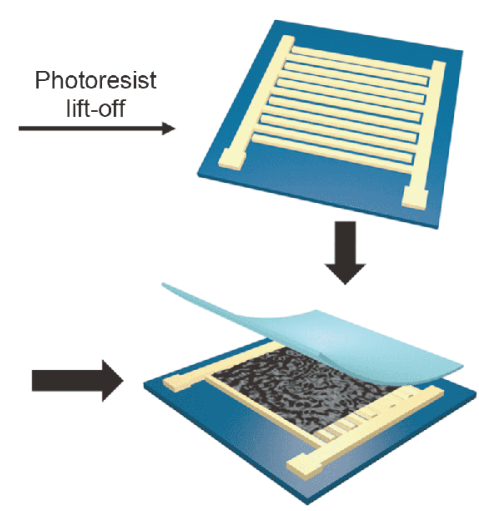

C

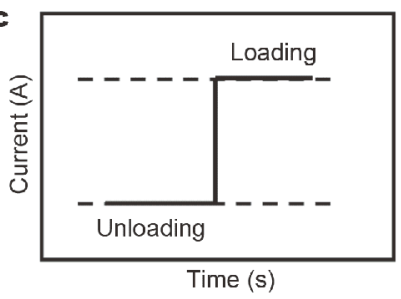

Figure 1 (a) Schematic illustration of the fabrication process of the pressure sensor. (b) Illustration of the material transformation with and without loading. (c) The current changes in response to loading and unloading 
sistance can return to the initial value due to the reduced contact area.

The PCI pressure sensor's working mechanism is composed of two parts, as described in Fig. 2. In the first stage, the connection resistance between the PBC film and $\mathrm{Cu}$ electrodes $\left(R_{\mathrm{C}}\right)$ is much larger than the resistance of the PBC film $\left(R_{\mathrm{F}}\right)$ itself [44]. Under small forces, the change in $R_{\mathrm{C}}$ is more obvious than the change in $R_{\mathrm{F}}$, which means $R_{\mathrm{C}}$ is a dominating factor affecting the variation in the pressure sensor resistance. As displayed in Fig. 2, the pressure sensor's initial total resistance $(R)$ can be expressed as follows:

$R=R_{\mathrm{F}}+R_{\mathrm{C}}+R_{\mathrm{M}}$,

where $R_{\mathrm{M}}$ is the resistance of the metal. $R_{\mathrm{C}}{ }^{\prime}$ can be calculated as follows:

$R_{\mathrm{C}}^{\prime}=R_{\mathrm{C}} / / R_{\mathrm{C}} / / \cdots \cdots / / R_{\mathrm{C}}$,

where $R_{\mathrm{C}}{ }^{\prime}$ means the change in $R_{\mathrm{C}}$ value. In the second stage, when the force is increased to a certain value, the contact area between the PBC film and the metal electrodes is stable. Subsequently, the internal change within the sensitive material plays a significant role in the variation of pressure sensor resistance. $\mathrm{PBC}$ fiber resistance $\left(R_{\mathrm{a}}, R_{\mathrm{b}}\right)$ is composed of the connected resistance of CNTs attached to the fiber. According to the two conductive fibers' distance from one another, the resistance can be divided into three cases [45]. There are three PBC fiber connection types, and their simplified circuit diagrams are displayed in Fig. S1. It is because of the two different mechanisms that the measurement range of the PCI pressure sensor may be divided into two linear regions.

Fig. 3a shows that the paper is composed of many spatially staggered fibers about $12 \mu \mathrm{m}$ in diameter, which can easily come in contact with each other when a load is applied. Because of the porous structure of the paper, the PBC film has a characteristically high specific surface area, so that the contact area of the fiber is easily altered under slightly changed pressure. Fig. 3b-d reveal that CNTs are evenly dispersed on the fiber surfaces, ensuring homogeneity of the PBC film. XRD and Raman spectroscopy were used to characterize the CNT composition, as displayed in Fig. 3e, f. As depicted in Fig. 3e, the diffraction peak of CNT is at $2 \theta=26^{\circ}$ (JCPDS: No.26-1080). The diffraction peak of CNT is sharp, indicating a high degree of crystallinity that engenders high conductivity. As shown in Fig. 3f, CNT has two obvious characteristic peaks "D" and "G". The D-band at $1350 \mathrm{~cm}^{-1}$ is related to the scattering of amorphous carbon. The G-band at around $1584 \mathrm{~cm}^{-1}$ presents the integrity of $\mathrm{sp}^{2}$ hybrid bond structures.

When a constant voltage was applied to the PCI pressure sensor, the initial $I$ and $R$ were acquired by measurements. The sensitivity, which is the essential factor reflecting the capability of the device, can be defined as follows:

$S=(\Delta I / I) / \Delta P$,

where $\Delta I$ is the change of current and $\Delta P$ represents variation in exerted pressure. Fig. 4 a shows the $I-V$ curves of the PCI pressure sensor with good linear ohmic characteristics, reflecting the device's stability with respect to static pressure. The sensor's slopes in the $I-V$ curves showed a clear decrease when the pressure increased from 0 to $39.2 \mathrm{kPa}$. The reason for the sensitive change in resistance is that the conductive paths are easily alerted. As shown in Fig. 4b, when the pressure changed across a wide measurement range from 0.7 to $39.2 \mathrm{kPa}$, the device retained high stability. Fig. $4 \mathrm{c}$ shows that the PCI pressure sensor displays a high sensitivity of $2.71 \mathrm{kPa}^{-1}$ and excellent linearity in the pressure range from 0 to $35 \mathrm{kPa}$. In the remaining pressure range from 35 to $140 \mathrm{kPa}$, the PCI pressure sensor displays a sensitivity of $0.21 \mathrm{kPa}^{-1}$. Furthermore, the two parts of the line indicate good coefficients of determination, with respective values 0.99 and 0.98 . We have prepared $\mathrm{PBC}$
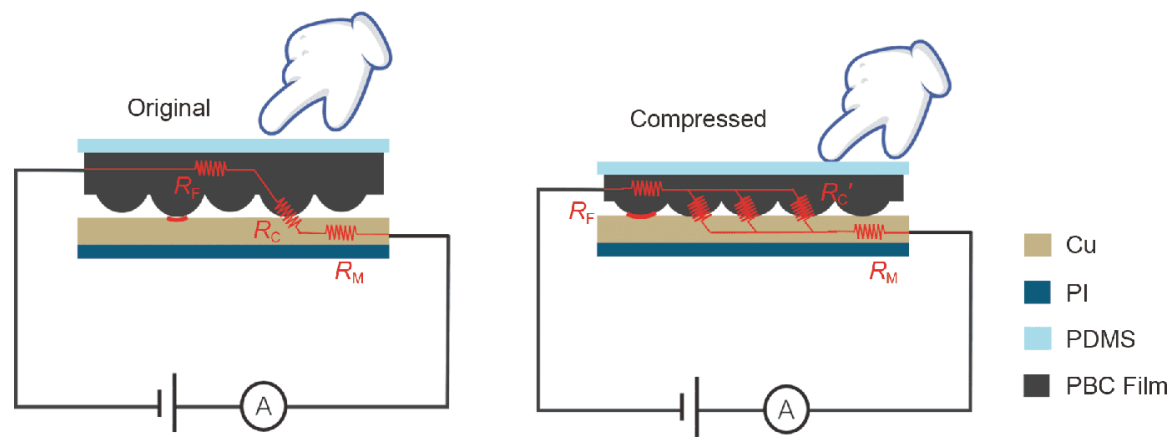

Figure 2 The sensing mechanism of PCI pressure sensor. 

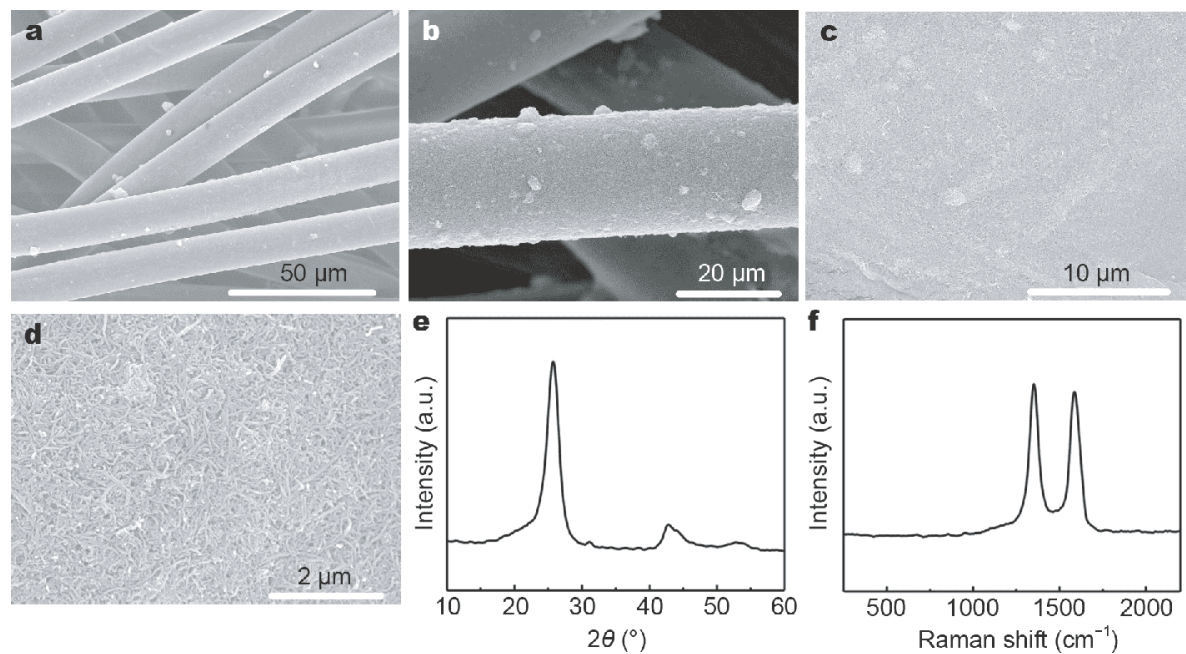

Figure 3 Microstructure and composition of the PBC film. (a-d) SEM images, (e) XRD and (f) Raman shift of the CNTs.
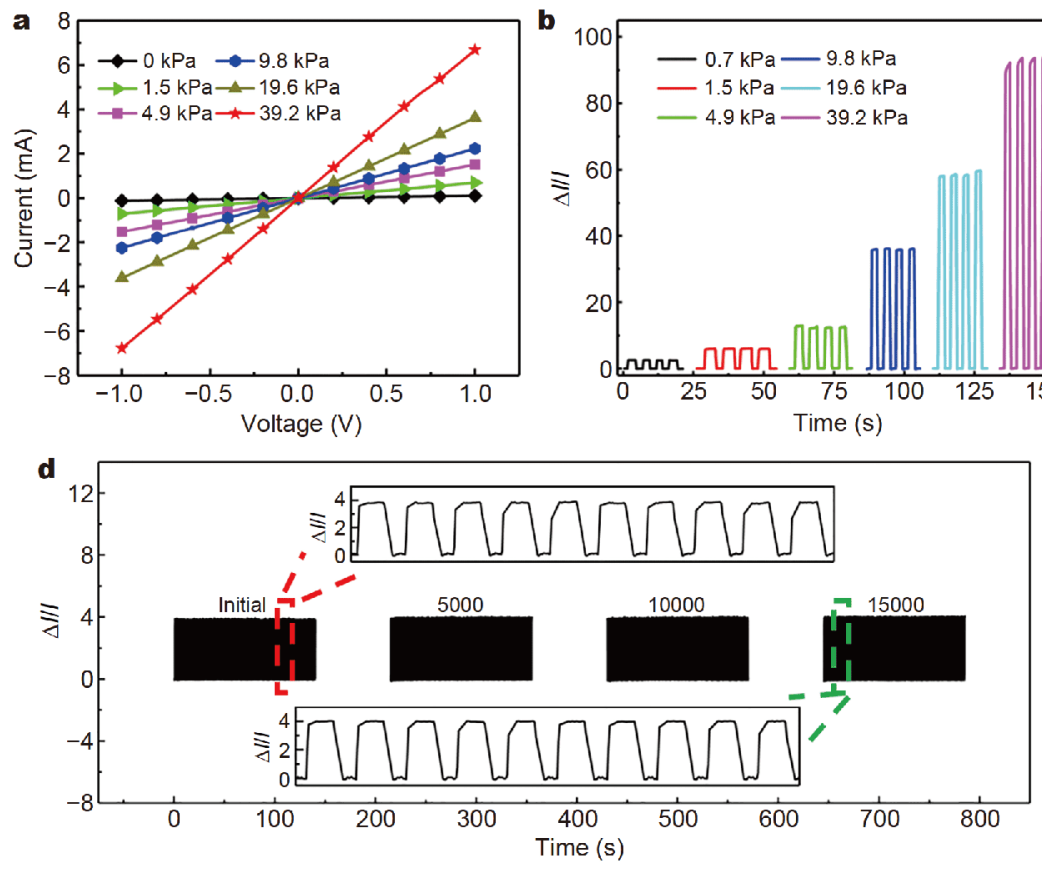
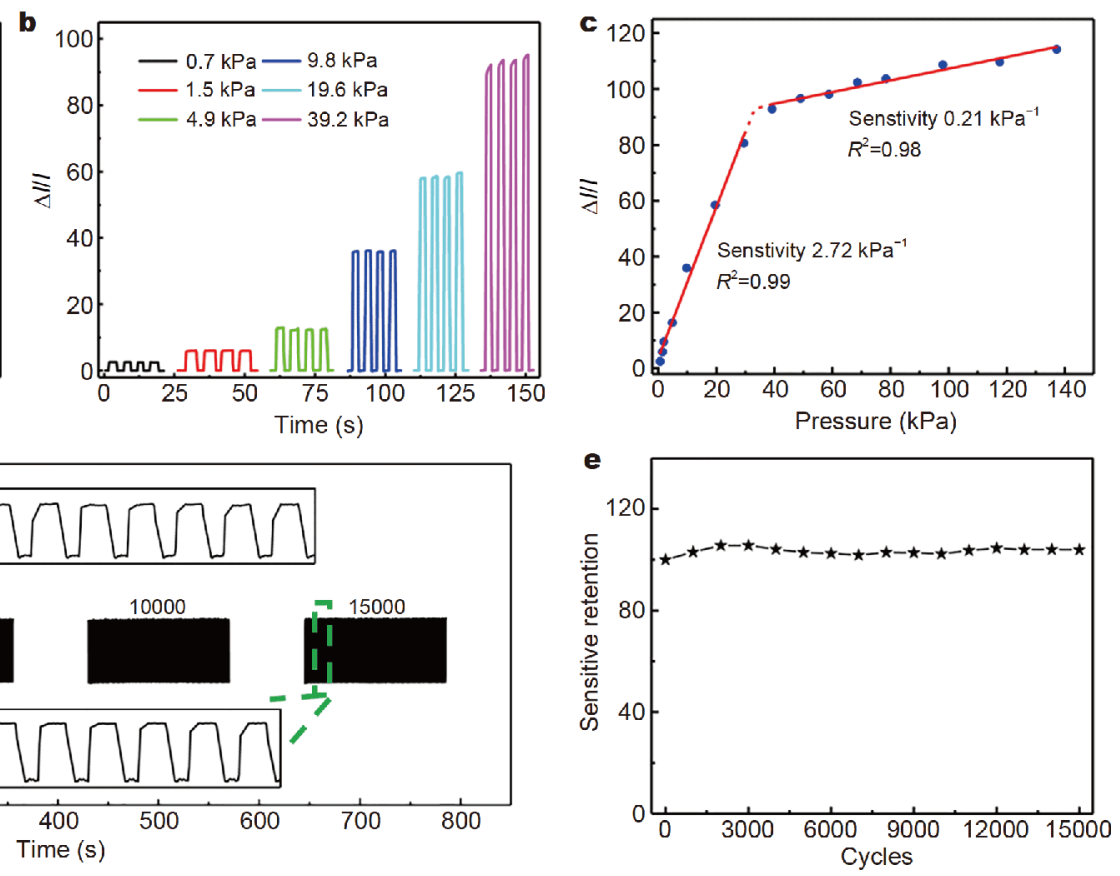

Figure 4 Properties of the pressure sensor. (a) $I$ - $V$ curves from -1 to $1 \mathrm{~V}$ under different pressures. (b) The change of current with various pressures applied. (c) The relationship between $\Delta I / I$ and $\Delta P$. (d) The stability of the pressure sensor after 15,000 cycles. (e) Sensitive retention.

films with different $\mathrm{CNT}$ concentrations ranging from $1 \%$ to $3 \%$, corresponding to pressure sensors $\mathrm{PBC} 1-\mathrm{PBC} 3$. The performance of the $\mathrm{PBC} 1$ and $\mathrm{PBC} 3$ pressure sensors in the pressure region from 0 to $10 \mathrm{kPa}$ was tested, while the $\mathrm{PBC} 2$ pressure sensor showed the best sensitivity (Fig. S2). The symbol " $\sim$ " means that the indicator is not mentioned in the literature. The pressure sensor's sensitivity is determined by the amplitude of resistance change in the device under a certain pressure, so a concentration of CNT that is too low or high produces conductive pathways in dearth or excess, leading to a small dynamic range in resistance. In Table 1, some pressure sensors with excellent performance are listed, including pressure range, sensitivity, and detection limit. Considering previously reported research, the broad measurement range of the present device is needed for practical applications. 
Table 1 Summary of partial pressure sensors

\begin{tabular}{|c|c|c|c|c|c|c|c|}
\hline Materials & $\begin{array}{l}\text { Upper pressure } \\
(\mathrm{kPa})\end{array}$ & $\begin{array}{l}\text { Linear range } \\
\qquad(\mathrm{kPa})\end{array}$ & $\begin{array}{l}\text { Sensitivity } \\
\left(\mathrm{kPa}^{-1}\right)\end{array}$ & $\begin{array}{l}\text { Detection } \\
\text { limit }(\mathrm{Pa})\end{array}$ & Stable cycles & $\begin{array}{c}\text { Working } \\
\text { voltage }(\mathrm{V})\end{array}$ & Ref. \\
\hline Gold nanowires & 5 & 5 & 1.14 & 13 & 50,000 & 1.5 & {$[46]$} \\
\hline Carbonaceous nanofibrous aerogels & 4.5 & 3 & 1.02 & 10 & 1000 & $\sim$ & {$[32]$} \\
\hline Suspended gate organic thin-film transistors & 5 & 5 & 192 & 0.5 & 100,000 & 60 & {$[47]$} \\
\hline Hollow-sphere-structured ppy & 10 & $\sim$ & $\sim$ & $\sim$ & 8000 & $\sim$ & {$[48]$} \\
\hline 3D graphene films & 75 & 0.2 & $130 / 3 / 0.26$ & 0.2 & 10,000 & 3 & {$[49]$} \\
\hline Carbonized silk nanofiber membrane & 5 & 0.4 & $34.47 / 1.16$ & 0.8 & 10,000 & $\sim$ & {$[50]$} \\
\hline Graphene & 25 & 25 & 1.2 & 5 & 6000 & 0.2 & {$[4]$} \\
\hline $\begin{array}{l}\text { Bubble-decorated honeycomb-like graphene } \\
\text { film }\end{array}$ & 10 & 0.56 & $161.6 / 0.92$ & 9 & 200 & 0.01 & {$[51]$} \\
\hline PPy@PVA-co-PE \& POE nanofiber & 7 & 0.15 & $1.24 / 0.89$ & 1.3 & $\sim$ & 2 & {$[31]$} \\
\hline AgNWs & 7.5 & 2 & $2.94 / 0.75$ & 3 & 1000 & $\sim$ & {$[52]$} \\
\hline CNT & 2.5 & 0.3 & $3.26 / 0.025$ & 45 & 5000 & $\sim$ & {$[20]$} \\
\hline $\mathrm{CNT}$ & 10 & 0.7 & $0.3 / 0.05$ & 20 & 5000 & $\sim$ & [53] \\
\hline CNT & 140 & 35 & $2.72 / 0.21$ & 8 & 15,000 & 2 & $\begin{array}{l}\text { This } \\
\text { work }\end{array}$ \\
\hline
\end{tabular}

The small linear region of the sensor means that a series of subsequent circuits are required for processing, such as amplifier circuits.

The present device exhibits very good repeatability over the course of 15,000 loading/unloading cycles, as depicted in Fig. 4d, e. Additionally, the initial and final waveform tests of the device showed almost the same results, and the response time of the pressure sensor was estimated to be $66 \mathrm{~ms}$ (Fig. S3). The PCI pressure sensor presented a better piezoresistive performance than the pressure sensor fabricated by dropping CNT solution directly onto the interdigitated electrodes (Fig. S4). When the CNT solution was dropped directly onto interdigitated electrodes to form the device, the slope of the $I-V$ curve representing device resistance did not change much under different pressures, since fewer conductive paths were formed. Due to the porous structure and anisotropy of the paper, the CNTs adsorbed on paper fibers could form many different conductive paths, allowing the slope of the $I-V$ curve to vary across broad pressures.

Wrist pulse is an essential physiological signal that reflects human health condition and can be detected precisely using wearable PCI pressure sensors. Fig. 5a, b show abundant information obtained from real-time wrist pulse signals of a healthy human wearing a flexible, noninvasive pulse sensor. Fig. $5 \mathrm{c}$ shows the details (parameters) of the wrist artery pulse, which includes percussion wave (P-wave), tidal wave (T-wave), and diastolic wave (D-wave). These important characteristics are extensively used in modern medicine to recognize the pulse process and are related to human health status. In medicine, the $\mathrm{P}$-wave is the first systolic blood pressure peak (SBP1) and represents the maximum point of aortic volume and vascular pressure in one artery pulse signal cycle [21]. The T-wave is the second systolic blood pressure peak (SBP2). It is called the heavy pre-wave and is related to the velocity of cardiovascular ejection, internal elasticity of arterial wall, tension and external resistance, as well as the interaction and resonance of various viscera and organs. Hence, the Augmentation Index (AI) can be calculated with the diastolic blood pressure (DBP) line using the expression $\mathrm{AI}=(\mathrm{SBP} 2-$ $\mathrm{DBP}) /(\mathrm{SBP} 1-\mathrm{DBP})$ to indicate human arterial stiffness [34]. Using a human volunteer, we estimated that the volunteer's pulse frequency was 75 beats/min with a regular and periodic waveform. As shown in Fig. 5d, the PCI pressure sensor was attached to the volunteer's neck for voice recognition. Fig. 5e shows that due to high sensitivity and flexibility of the device, diverse characteristic signals could be displayed when the volunteer said various words such as "Hello", "Success", and "Nature". In addition, the same characteristic signals were displayed repeatedly three times when the volunteer repeated the word "Nature", demonstrating the high reliability and repeatability of the device, as shown in Fig. $5 f$. Movement of throat muscles is expected to be essentially identical when different volunteers speak the same word, so similar pressures are expected to be experienced by the device. The device is applicable not only in rehabilitation courses for voice practice but also as a remote control for 


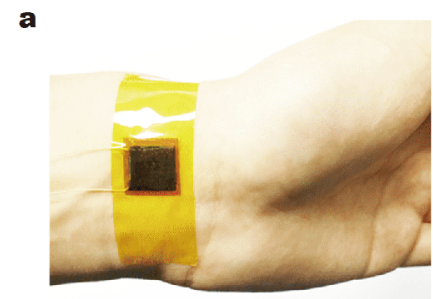

d
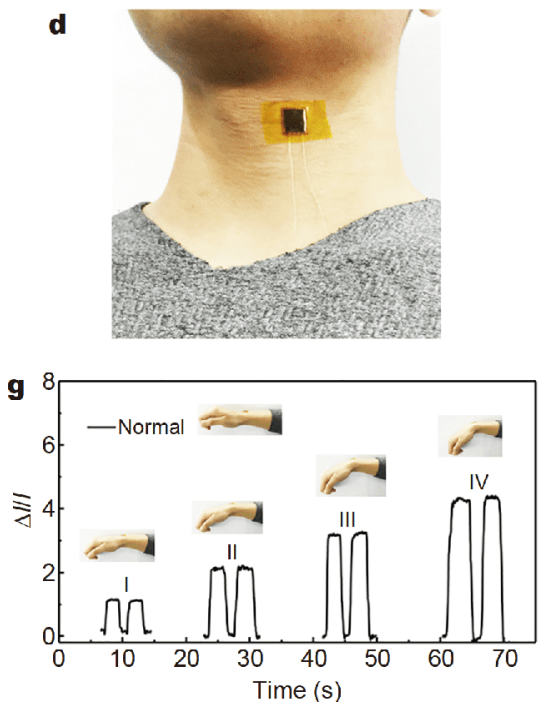

b

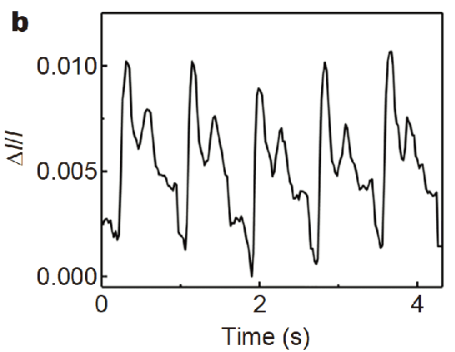

e
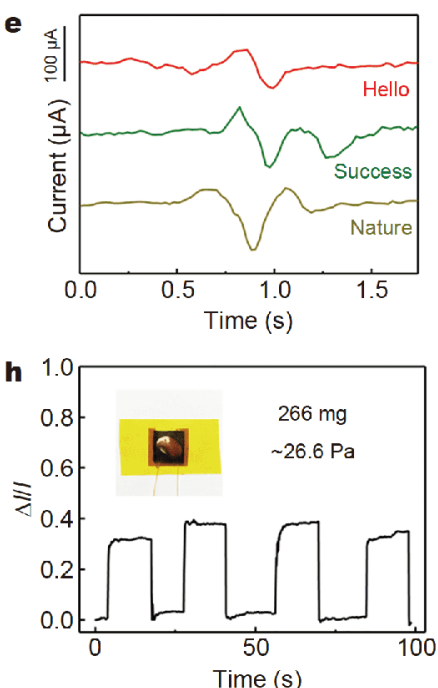
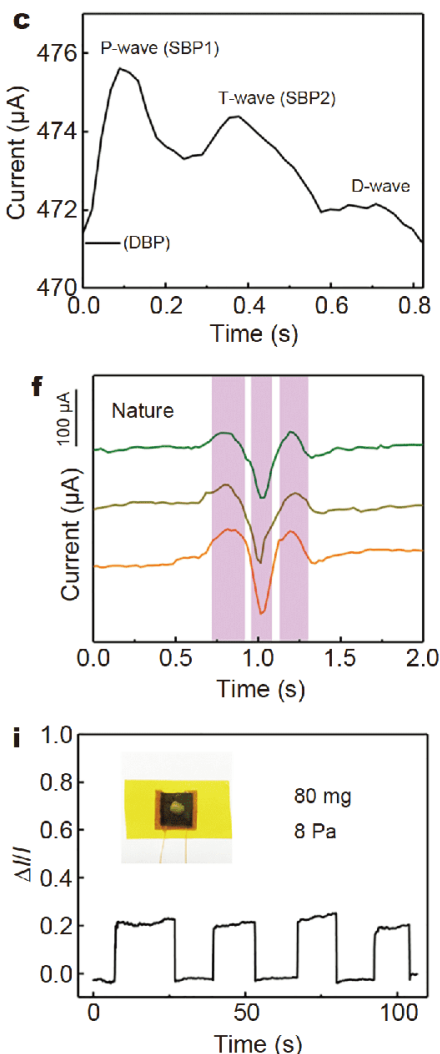

Figure 5 The flexible pressure sensor for various applications. (a) Photograph of the pressure sensor attached at the wrist as a pulse sensor. (b) The record of the pulse wave. (c) The amplified waveform from (b). (d) Photograph of the pressure sensor attached to the neck. (e) The different waveforms of the various words. (f) Same word has the same characteristic of the waveform. (g) Monitoring of wrist movement. Real-time currenttime (I-t) curves of (h) mung bean and (i) red bean, respectively.

machines. As shown in Fig. 5g, the pressure sensor used in detecting human posture was attached to the wrist of the researcher, whose bending angle changed and then stayed in that position for a few seconds. In Fig. 5h, i, the weights of the mung bean and red bean, about $80 \mathrm{mg}$ $(\sim 8 \mathrm{~Pa})$ and $266 \mathrm{mg}(\sim 26.6 \mathrm{~Pa})$, respectively, were sensitively discerned by the PCI pressure sensor. When different pressures were applied to the PCI pressure sensor in series with an LED lamp, the brightness of the LED lamp changed with the change in the sensor's resistance (Fig. S5).

In order to improve human-machine interaction and Eskin technology, high-performance pressure sensor arrays are in urgent demand [54]. Fig. 6a, b show the flexible pressure sensor arrays used to detect spatial pressure distribution, containing $3 \times 3$ pixels and an area of $10 \mathrm{~mm}$ $\times 10 \mathrm{~mm}$. Two weights $(2,5 \mathrm{~g})$ were placed on different pixels of the array, causing a change in the spatial pressure distribution and thus to the resistance, as shown in Fig. 6c. Other future applications are plentiful for PCI pressure sensor arrays used as human-machine interactive interfaces for real-time spatial pressure distribution analysis. Fig. 6d, e show the pixel resistances of the array being differently and sequentially altered when the letter "L" was being written on the array. In conclusion, the $3 \mathrm{D}$ space that contains time, position, and strength can be well reflected through a high-reliability piezoresistive sensor array.

\section{CONCLUSIONS}

A pressure sensor with simultaneously high sensitivity and wide measurement range was proposed based on PCI electrodes, then constructed and tested. The flexible sensor reliably measured pressure with a sensitivity of $2.72 \mathrm{kPa}^{-1}$ in the range of 0 to $35 \mathrm{kPa}$ and $0.21 \mathrm{kPa}^{-1}$ within the range of 0 to $140 \mathrm{kPa}$. After 15,000 loading and unloading cycles, the pressure sensor exhibited outstanding reliability and stability. Sensitively detecting pressure sensor exerted by an $80 \mathrm{mg}$ resting mung bean ( $\sim \mathrm{Pa})$, this pressure sensor shows promise for applica- 
a
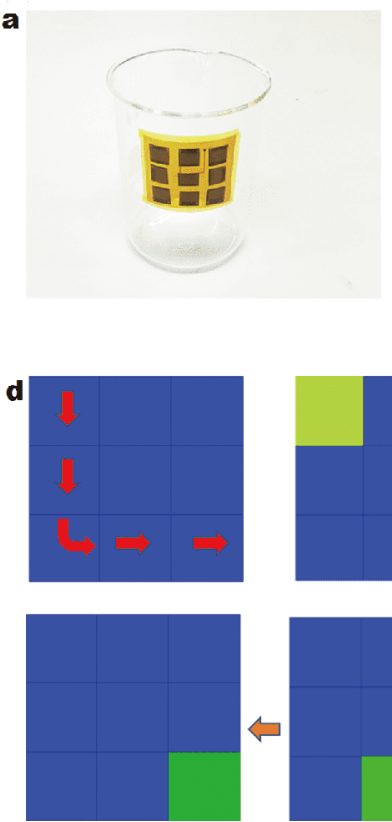

b
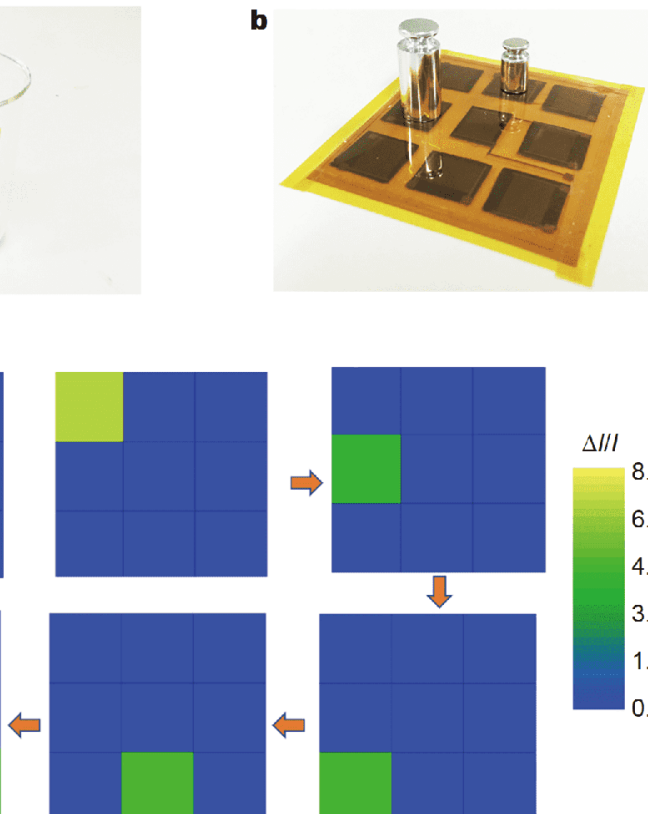

c
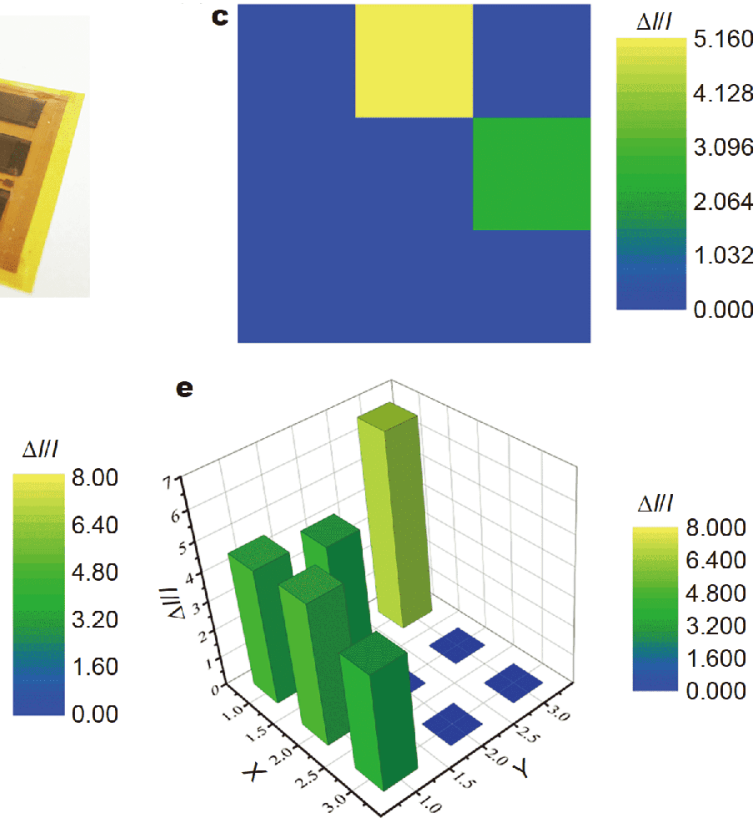

e

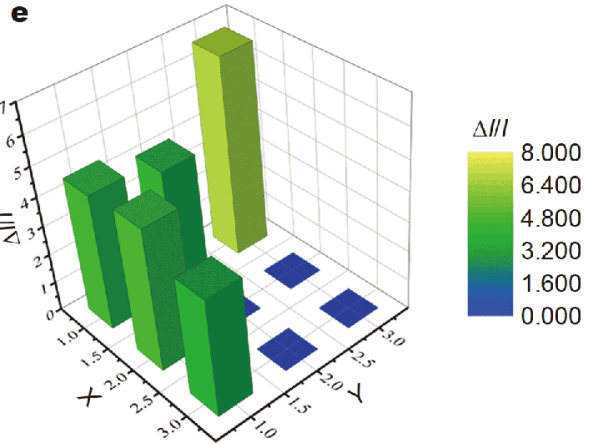

Figure 6 (a) The flexible pressure sensor array attached to the beaker. (b) Photograph of two different weights on the flexible pressure sensor array. (c) Measurement of the spatial pressure distribution. (d, e) The measurement of the dynamic pressure while the letter "L" was written.

tions in wrist pulse detection, voice recognition, and other human movement posture monitoring. In addition, this pressure sensor exhibits potential in the field of human-machine interaction interfaces and E-skin via application in large-area sensors to discern spatial static and dynamic pressure distributions. The high sensitivity, wide measurement range, and low cost of such pressure sensors recommend their extensive use in wearable devices.

Received 23 July 2019; accepted 28 August 2019;

published online 9 October 2019

1 Niu X, Jia W, Qian S, et al. High-performance PZT-based stretchable piezoelectric nanogenerator. ACS Sustain Chem Eng, 2019, 7: 979-985

2 Lou Z, Li L, Wang L, et al. Recent progress of self-powered sensing systems for wearable electronics. Small, 2017, 13: 1701791

3 Chun S, Son W, Choi C. Flexible pressure sensors using highlyoriented and free-standing carbon nanotube sheets. Carbon, 2018, 139: 586-592

4 Shi J, Wang L, Dai Z, et al. Multiscale hierarchical design of a flexible piezoresistive pressure sensor with high sensitivity and wide linearity range. Small, 2018, 14: 1800819

5 Bae GY, Pak SW, Kim D, et al. Linearly and highly pressuresensitive electronic skin based on a bioinspired hierarchical structural array. Adv Mater, 2016, 28: 5300-5306

6 Wang R, Xu LH, Li YQ, et al. Broadband light out-coupling enhancement of flexible organic light-emitting diodes using biomimetic quasirandom nanostructures. Adv Opt Mater, 2015, 3: 203210
7 Zhao L, Zhang L, Zhao J, et al. Engineering surface patterns with shape memory polymers: Multiple design dimensions for diverse and hierarchical structures. ACS Appl Mater Interfaces, 2018, 11: 1563-1570

8 Shi R, Lou Z, Chen S, et al. Flexible and transparent capacitive pressure sensor with patterned microstructured composite rubber dielectric for wearable touch keyboard application. Sci China Mater, 2018, 61: 1587-1595

9 Kwon D, Lee TI, Shim J, et al. Highly sensitive, flexible, and wearable pressure sensor based on a giant piezocapacitive effect of three-dimensional microporous elastomeric dielectric layer. ACS Appl Mater Interfaces, 2016, 8: 16922-16931

10 Chen Z, Wang Z, Li X, et al. Flexible piezoelectric-induced pressure sensors for static measurements based on nanowires/graphene heterostructures. ACS Nano, 2017, 11: 4507-4513

11 Shin KY, Lee JS, Jang J. Highly sensitive, wearable and wireless pressure sensor using free-standing $\mathrm{ZnO}$ nanoneedle/PVDF hybrid thin film for heart rate monitoring. Nano Energy, 2016, 22: 95-104

12 Gong $\mathrm{T}$, Zhang $\mathrm{H}$, Huang $\mathrm{W}$, et al. Highly responsive flexible strain sensor using polystyrene nanoparticle doped reduced graphene oxide for human health monitoring. Carbon, 2018, 140: 286-295

13 Song $\mathrm{Y}$, Chen $\mathrm{H}, \mathrm{Su} \mathrm{Z}$, et al. Highly compressible integrated supercapacitor-piezoresistance-sensor system with CNT-PAMS sponge for health monitoring. Small, 2017, 13: 1702091

14 Chen X, Song Y, Chen $\mathrm{H}$, et al. An ultrathin stretchable triboelectric nanogenerator with coplanar electrode for energy harvesting and gesture sensing. J Mater Chem A, 2017, 5: 1236112368

15 Chen $\mathrm{T}$, Zhao M, Shi Q, et al. Novel augmented reality interface using a self-powered triboelectric based virtual reality $3 \mathrm{D}$-control sensor. Nano Energy, 2018, 51: 162-172 
16 Dong K, Deng J, Ding W, et al. Versatile core-sheath yarn for sustainable biomechanical energy harvesting and real-time humaninteractive sensing. Adv Energy Mater, 2018, 8: 1801114

17 Shi J, Li X, Cheng H, et al. Graphene reinforced carbon nanotube networks for wearable strain sensors. Adv Funct Mater, 2016, 26: 2078-2084

18 Lee JW, Chung J, Cho MY, et al. A deep-learning technique to convert a crude piezoresistive CNT-Ecoflex composite sheet into a smart, portable, disposable, and extremely flexible keypad. ACS Appl Mater Interfaces, 2018, 10: 20862-20868

19 Liu W, Liu N, Yue Y, et al. A flexible and highly sensitive pressure sensor based on elastic carbon foam. J Mater Chem C, 2018, 6: 1451-1458

20 Cao Y, Li T, Gu Y, et al. Fingerprint-inspired flexible tactile sensor for accurately discerning surface texture. Small, 2018, 14: 1703902

21 Wang X, Gu Y, Xiong Z, et al. Silk-molded flexible, ultrasensitive, and highly stable electronic skin for monitoring human physiological signals. Adv Mater, 2014, 26: 1336-1342

22 Liang B, Chen W, He Z, et al. Highly sensitive, flexible MEMS based pressure sensor with photoresist insulation layer. Small, 2017, 13: 1702422

23 Gerratt AP, Michaud HO, Lacour SP. Elastomeric electronic skin for prosthetic tactile sensation. Adv Funct Mater, 2015, 25: 22872295

24 Cho SH, Lee SW, Yu S, et al. Micropatterned pyramidal ionic gels for sensing broad-range pressures with high sensitivity. ACS Appl Mater Interfaces, 2017, 9: 10128-10135

25 Chang H, Kim S, Jin S, et al. Ultrasensitive and highly stable resistive pressure sensors with biomaterial-incorporated interfacial layers for wearable health-monitoring and human-machine interfaces. ACS Appl Mater Interfaces, 2018, 10: 1067-1076

26 Liu W, Liu N, Yue Y, et al. Piezoresistive pressure sensor based on synergistical innerconnect polyvinyl alcohol nanowires/wrinkled graphene film. Small, 2018, 14: 1704149

27 Chen W, Gui X, Liang B, et al. Structural engineering for high sensitivity, ultrathin pressure sensors based on wrinkled graphene and anodic aluminum oxide membrane. ACS Appl Mater Interfaces, 2017, 9: 24111-24117

28 Jian M, Xia K, Wang Q, et al. Flexible and highly sensitive pressure sensors based on bionic hierarchical structures. Adv Funct Mater, 2017, 27: 1606066

29 Lee J, Kwon H, Seo J, et al. Conductive fiber-based ultrasensitive textile pressure sensor for wearable electronics. Adv Mater, 2015, 27: 2433-2439

30 Fu X, Dong H, Zhen Y, et al. Solution-processed large-area nanocrystal arrays of metal-organic frameworks as wearable, ultrasensitive, electronic skin for health monitoring. Small, 2015, 11: 3351-3356

31 Zhong W, Liu Q, Wu Y, et al. A nanofiber based artificial electronic skin with high pressure sensitivity and $3 \mathrm{D}$ conformability. Nanoscale, 2016, 8: 12105-12112

32 Si Y, Wang X, Yan C, et al. Ultralight biomass-derived carbonaceous nanofibrous aerogels with superelasticity and high pressuresensitivity. Adv Mater, 2016, 28: 9512-9518

33 Chen S, Lou Z, Chen D, et al. Highly flexible strain sensor based on $\mathrm{ZnO}$ nanowires and $\mathrm{P}$ (VDF-TRFE) fibers for wearable electronic device. Sci China Mater, 2016, 59: 173-181

34 Wang Z, Wang S, Zeng J, et al. High sensitivity, wearable, piezoresistive pressure sensors based on irregular microhump structures and its applications in body motion sensing. Small, 2016, 12: 3827-
3836

35 Souri $\mathrm{H}, \mathrm{Yu}$ J, Jeon $\mathrm{H}$, et al. A theoretical study on the piezoresistive response of carbon nanotubes embedded in polymer nanocomposites in an elastic region. Carbon, 2017, 120: 427-437

36 Wang M, Zhang K, Dai XX, et al. Enhanced electrical conductivity and piezoresistive sensing in multi-wall carbon nanotubes/polydimethylsiloxane nanocomposites via the construction of a selfsegregated structure. Nanoscale, 2017, 9: 11017-11026

37 Song $\mathrm{Y}$, Chen $\mathrm{H}$, Chen $\mathrm{X}$, et al. All-in-one piezoresistive-sensing patch integrated with micro-supercapacitor. Nano Energy, 2018, 53: $189-197$

38 Choong CL, Shim MB, Lee BS, et al. Highly stretchable resistive pressure sensors using a conductive elastomeric composite on a micropyramid array. Adv Mater, 2014, 26: 3451-3458

39 Wang L, Wang K, Lou Z, et al. Plant-based modular building blocks for "green" electronic skins. Adv Funct Mater, 2018, 28: 1804510

40 Iglio R, Mariani S, Robbiano V, et al. Flexible polydimethylsiloxane foams decorated with multiwalled carbon nanotubes enable unprecedented detection of ultralow strain and pressure coupled with a large working range. ACS Appl Mater Interfaces, 2018, 10: 13877-13885

41 Huang W, Dai K, Zhai Y, et al. Flexible and lightweight pressure sensor based on carbon nanotube/thermoplastic polyurethanealigned conductive foam with superior compressibility and stability. ACS Appl Mater Interfaces, 2017, 9: 42266-42277

42 Wang $\mathrm{X}$, Dong $\mathrm{L}$, Zhang $\mathrm{H}$, et al. Recent progress in electronic skin. Adv Sci, 2015, 2: 1500169

43 Shin SH, Ji S, Choi S, et al. Integrated arrays of air-dielectric graphene transistors as transparent active-matrix pressure sensors for wide pressure ranges. Nat Commun, 2017, 8: 14950

44 Luo N, Dai W, Li C, et al. Flexible piezoresistive sensor patch enabling ultralow power cuffless blood pressure measurement. Adv Funct Mater, 2016, 26: 1178-1187

45 Amjadi M, Pichitpajongkit A, Lee S, et al. Highly stretchable and sensitive strain sensor based on silver nanowire-elastomer nanocomposite. ACS Nano, 2014, 8: 5154-5163

46 Gong S, Schwalb W, Wang Y, et al. A wearable and highly sensitive pressure sensor with ultrathin gold nanowires. Nat Commun, 2014, 5: 3132

47 Pan L, Chortos A, Yu G, et al. An ultra-sensitive resistive pressure sensor based on hollow-sphere microstructure induced elasticity in conducting polymer film. Nat Commun, 2014, 5: 3002

48 Zang Y, Zhang F, Huang D, et al. Flexible suspended gate organic thin-film transistors for ultra-sensitive pressure detection. Nat Commun, 2015, 6: 6269

49 Xia K, Wang C, Jian M, et al. CVD growth of fingerprint-like patterned 3D graphene film for an ultrasensitive pressure sensor. Nano Res, 2017, 11: 1124-1134

50 Wang Q, Jian M, Wang C, et al. Carbonized silk nanofiber membrane for transparent and sensitive electronic skin. Adv Funct Mater, 2017, 27: 1605657

51 Sheng L, Liang Y, Jiang L, et al. Bubble-decorated honeycomb-like graphene film as ultrahigh sensitivity pressure sensors. Adv Funct Mater, 2015, 25: 6545-6551

52 Shuai X, Zhu P, Zeng W, et al. Highly sensitive flexible pressure sensor based on silver nanowires-embedded polydimethylsiloxane electrode with microarray structure. ACS Appl Mater Interfaces, 2017, 9: 26314-26324

53 Kim KH, Hong SK, Jang NS, et al. Wearable resistive pressure 
sensor based on highly flexible carbon composite conductors with irregular surface morphology. ACS Appl Mater Interfaces, 2017, 9: 17499-17507

54 Hammock ML, Chortos A, Tee BCK, et al. 25th anniversary article: The evolution of electronic skin (e-skin): A brief history, design considerations, and recent progress. Adv Mater, 2013, 25: 59976038

Acknowledgements The authors acknowledge the funding support from the National Natural Science Foundation of China (51605449, 51675493 and 51705476), and Shanxi "1331 Project" Key Subject Construction (1331KSC).

Author contributions Wang $\mathrm{C}$ and Hou $\mathrm{X}$ designed the devices and experiments; Wang C, Hou X, Cui M, Yu J, and He J performed the experiments; Wang C, Fan X, Qian J, Geng W, and Mu J analyzed the data; Wang $\mathrm{C}$ wrote the paper with support from Hou X and Chou X. All authors contributed to the general discussion.

Conflict of interest There are no conflicts to declare.

Supplementary information Supporting data are available in the online version of the paper.

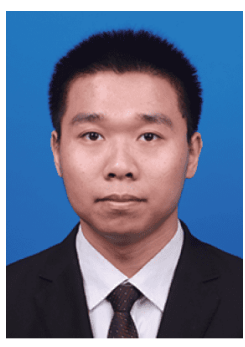

Chao Wang received his BSc degree from North University of China (NUC) in 2016. Now he is a graduate student at the NUC. His research interest focuses on flexible pressure sensor and wearable electronic devices.

\section{纸基碳纳米管薄膜/叉指结构的超灵敏宽量程压 力传感器}

王超, 侯晓娟, 崔敏, 余俊斌, 范雪明, 钱冀超, 何剑, 耿文平, 穆继亮, 丑修建

摘要 柔性压力传感器因其在可穿戴设备和人机交互界面中的潜 在应用而备受关注. 特别是在实际应用中, 人们对具有高灵敏度、 宽测量范围和低成本的压力传感器有很大需求. 基于此, 我们研制 出了一种测量范围宽的超灵敏压力传感器. 该传感器是以碳纳米 管 (CNT) 均匀溶液直接喷在纸表面作为敏感材料, 用光刻技术制成 的叉指电极为结构. 由于 CNT大的比表面积、纸的多孔结构以及 $\mathrm{CNT}$ 与叉指电极有效接触的协同作用, 压力传感器实现了从 0 到 $140 \mathrm{kPa}$ 的宽测量范围, 并在 15,000 个测试周期内表现出良好的稳 定性. 对于纸基碳纳米管薄膜/叉指状结构(PCI)压力传感器, 敏感 材料与叉指电极之间的连接区域在较小的压力范围内占主导地位, 而敏感材料的内部变化在大的压力区域起主导作用. 此外PCI压力 传感器不仅具有 $2.72 \mathrm{kPa}^{-1}$ (直到 $35 \mathrm{kPa}$ ) 的高灵敏度, 还可以检测小 重量, 如一颗绿豆 (约 $8 \mathrm{~Pa}$ ). 当压力传感器贴附到人体表面时, 可以 监测生理信号, 如手腕运动、脉搏跳动和语音识别. 此外, 压力传感 器的阵列能够识别空间压力分布, 有望实际应用于人机交互界面.

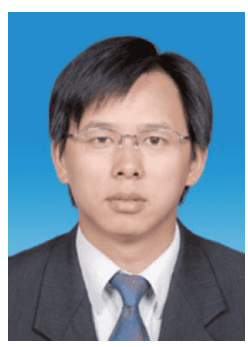

Xiujian Chou works at the School of Instrument and Electronics of NUC. He received his $\mathrm{PhD}$ degree in material physics and chemistry at Tongji University in 2008. Currently he is engaged in intelligent micro/nano device and micro system. 